\title{
INVERSE MULTIPARAMETER EIGENVALUE PROBLEMS FOR MATRICES II
}

\author{
by PATRICK J. BROWNE* and B. D. SLEEMAN \\ (Received 11th February 1985)
}

\section{Introduction}

This is a sequel to our previous paper [4] where we initiated a study of inverse eigenvalue problems for matrices in the multiparameter setting. The one parameter version of the problem under consideration asks for conditions on a given $n \times n$ symmetric matrix $A$ and on $n$ given real numbers $s_{1} \leqq s_{2} \leqq \cdots \leqq s_{n}$ under which a diagonal matrix $V$ can be found so that $A+V$ has $s_{1}, \ldots, s_{n}$ as its eigenvalues. Our motivation for this problem and our method of attack on it in [4] comes chiefly from the work of Hadeler [5] in which sufficient conditions were given for existence of the desired diagonal $V$. Hadeler's approach in [5] relied heavily on the Brouwer fixed point theorem and this was also our main tool in [4]. Subsequently, using properties of topological degree, Hadeler [6] gave somewhat different conditions for the existence of the diagonal $V$. It is our desire here to follow this lead and to use degree theory to give some results extending those in [6] to the multiparameter case.

In Section 2 we study the inverse eigenvalue problem for one equation with two spectral parameters and in Section 3 we apply these results to linked systems of such equations and to the quadratic eigenvalue problem thus paralleling our earlier work [4].

\section{One equation with two parameters}

In this section we are given $n \times n$ symmetric matrices $A, B, C$ where, without loss of generality, we assume that the leading diagonal elements of $A$ namely $a_{i i}=0,1 \leqq i \leqq n$. For each $(\lambda, \mu) \in \mathbb{R}^{2}$ the matrix

$$
W(\lambda, \mu)=A+\lambda B+\mu C
$$

is also symmetric and we list its eigenvalues as

$$
\rho_{1}(A ; \lambda, \mu) \leqq \cdots \leqq \rho_{n}(A ; \lambda, \mu)
$$

* Research supported in part by the NSERC of Canada and The University of Dundee. 
We are interested in the eigencurves given by

$$
Z_{i}(A)=\left\{(\lambda, \mu) \in \mathbb{R}^{2} \mid \rho_{i}(A ; \lambda, \mu)=0\right\}
$$

There is no a priori guarantee that the sets $Z_{i}(A)$ are nonempty but various fairly weak conditions preventing $Z_{i}(A)=\varnothing$ have been discussed in [2]. It will be enough for us here to assume that at least one of $B, C$ is positive (or negative) definite.

As in [4], we use the cone $\hat{C} \subset \mathbb{R}^{2}$ given by

$$
\widehat{C}=\left\{(\lambda, \mu) \mid \lambda(B x, x)+\mu(C x, x) \leqq 0, \forall x \in \mathbb{R}^{n}\right\}
$$

and we assume

Hypothesis 2.1. The points $\left(s_{i}, t_{i}\right)$ are $\hat{C}$-ordered; i.e.

$$
\left(s_{j}, t_{j}\right)-\left(s_{i}, t_{i}\right) \in \hat{C} \text { whenever } j \geqq i .
$$

We put

$$
g_{i}^{j}=\sum_{\substack{k=1 \\ k \neq i}}^{n}\left|a_{i k}+s_{j} b_{i k}+t_{j} c_{i k}\right|
$$

and make the further

\section{Hypothesis 2.2.}

$$
\begin{gathered}
\left(s_{j}-s_{i}\right) b_{i i}+\left(t_{j}-t_{i}\right) c_{i i}<-g_{i}^{j}-g_{j}^{j}, \\
\left(s_{k}-s_{j}\right) b_{k k}+\left(t_{k}-t_{j}\right) c_{k k}<-g_{k}^{j}-g_{j}^{j},
\end{gathered}
$$

$$
1 \leqq i<j<k \leqq n .
$$

Note that Hypothesis 2.1 ensures that the left-hand sides of these two inequalities are, in fact, negative. Now select $\eta>0$ and consider the open bounded region $E \subset \mathbb{R}^{n}$ given by

$$
\begin{array}{r}
E=\left\{\left(v_{1}, \ldots, v_{n}\right) \mid v_{1}+s_{1} b_{11}+t_{1} c_{11}>-\eta, v_{n}+s_{n} b_{n n}+t_{n} c_{n n}<\eta,\right. \\
v_{i}+s_{j} b_{i i}+t_{j} c_{i i}+g_{i}^{j}<v_{j}+s_{j} b_{j j}+t_{j} c_{j j}-g_{j}^{j}-\varepsilon, \\
v_{j}+s_{j} b_{j j}+t_{j} c_{j j}+g_{j}^{j}<v_{k}+s_{j} b_{k k}+t_{j} c_{k k}-g_{k}^{j}-\varepsilon, \\
1 \leqq i<j<k \leqq n\} .
\end{array}
$$

It is easy to check that the point

$$
x=\left(-s_{1} b_{11}-t_{1} c_{11}, \ldots,-s_{n} b_{n n}-t_{n} c_{n n}\right)
$$

belongs to $E$. 
For $v \in E, V$ will denote the diagonal matrix $V=\operatorname{diag}\left(v_{1}, \ldots, v_{n}\right)$. We also use $\hat{B}, \hat{C}$ to denote the diagonal matrices

$$
\hat{B}=\operatorname{diag}\left(b_{11}, \ldots, b_{n n}\right), \quad \hat{C}=\operatorname{diag}\left(c_{11}, \ldots, c_{n n}\right)
$$

and $B^{\#}, C^{\#}$ for $B-\hat{B}, C-\hat{C}$ respectively. Now consider the mapping $F_{\theta}, 0 \leqq \theta \leqq 1$, $F_{\theta}: E \rightarrow \mathbb{R}^{n}$ given by

$$
\begin{aligned}
F_{\theta}(v)= & \left(\rho_{1}\left(\theta\left(A+s_{1} B^{\#}+t_{1} C^{\#}\right)+V+s_{1} \hat{B}+t_{1} \hat{C}\right),\right. \\
& \left.\rho_{n}\left(\theta\left(A+s_{n} B^{*}+t_{n} C^{*}\right)+V+s_{n} \hat{B}+t_{n} \hat{C}\right)\right) .
\end{aligned}
$$

Our problem of finding a diagonal matrix $V$ so that $\left(s_{i}, t_{i}\right) \in Z_{i}(A+V), 1 \leqq i \leqq n$, is equivalent to finding a point $v$ so that $F_{1}(v)=0$.

Note that for $v \in E$,

$$
\begin{aligned}
& v_{i}+s_{j} b_{i i}+t_{j} c_{i i}<v_{j}+s_{j} b_{j j}+t_{j} c_{j j}<v_{k}+s_{j} b_{k k}+t_{j} c_{k k}, \\
& 1 \leqq i<j<k \leqq n .
\end{aligned}
$$

Thus it follows that

$$
F_{0}(v)=v+x
$$

and accordingly $F_{0}(v)=0$ has a unique solution, viz. $v=-x$. Moreover, in terms of the topological degree we see that

$$
d\left(F_{0}, E, 0\right)=1
$$

It is clear that $F_{0}$ and $F_{1}$ are homotopy equivalent. To use the homotopy invariance of topological degree we need to show that for each $\theta \in[0,1]$ we have $0 \notin F_{\theta}(\partial E)$. Suppose then that $v \in \partial E$ and $F_{\theta}(v)=0$. Should $v \in \partial E$, because $v_{1}+s_{11} b_{11}+t_{1} c_{11}=-\eta$, we can argue that $W\left(A+V ; s_{1}, t_{1}\right)$ is positive semi-definite (since zero is its smallest eigenvalue) and thus its diagonal entries must be non-negative. Hence $v_{1}+s_{1} b_{11}+t_{1} c_{11} \geqq 0$-a contradiction. In like fashion we can dismiss the case $v_{n}+s_{n} b_{n n}+t_{n} c_{n n}=\eta$. We next note that the matrix $\theta\left(A+s_{j} B^{\#}+t_{j} C^{\#}\right)+V+s_{j} \hat{B}+t_{j} \hat{C}$ has diagonal entries $v_{i}+s_{j} b_{i i}+t_{j} c_{i i}$, $1 \leqq i \leqq n$, which are the centres of the Gerschgorin circles for this matrix. The radii of the circles are $\theta g_{i}^{j}, 1 \leqq i \leqq n$, respectively. From the relations defining $E$ we see that the circles corresponding to $i=1, \ldots, j-1$ are all disjoint from the $j$ th circle which in turn is disjoint from the circles corresponding to $i=j+1, \ldots, n$. We use the theorems of Hadamard and Gerschgorin (see [1, Theorems 6.2.1, 6.2.2, p. 231]) to infer that the $j$ th circle contains $\rho_{j}\left(\theta\left(A+s_{j} B^{\#}+t_{j} C^{\#}\right)+V+s_{j} \hat{B}+t_{j} \hat{C}\right)$ and thus if $F_{\theta}(v)=0$ we must have $\left|v_{j}+s_{j} b_{j j}+t_{j} c_{j j}\right| \leqq \theta g_{j}^{j}$. This observation is now sufficient to complete the proof that $F_{\theta}(v) \neq 0$ for $v \in \partial E$.

The upshot of these remarks is

Theorem 1. Suppose Hypothesis 2.2 holds. Then there is a diagonal $V=$ 
$\operatorname{diag}\left(v_{1}, \ldots, v_{n}\right)$ such that

$$
\left(s_{i}, t_{i}\right) \in Z_{i}(A+V)
$$

and

$$
\left|v_{i}+s_{i} b_{i i}+t_{i} c_{i i}\right| \leqq g_{i}^{i}, \quad 1 \leqq i \leqq n
$$

Theorem 2. The conclusion of Theorem 1 holds if equality is permitted in the two inequalities of Hypothesis 2.2 .

Proof. If $g_{j}^{j} \neq 0$ for each $1 \leqq j \leqq n$ then the argument above shows that for each $\theta \in[0,1)$ we have a solution $v^{\theta}$ of $F_{\theta}(v)=0$. We select a sequence $\theta_{k} \rightarrow 1$ with corresponding solutions $v^{k}$. A suitable subsequence of $v^{k}$ must converge and the limit will be a solution of $F_{1}(v)=0$. Whenever $g_{j}^{j}=0$ it is easy to see that it is necessary to use $v_{j}=-s_{j} b_{j j}-t_{j} c_{j j}$

We should point out that while we have considered here an equation with exactly two parameters, similar arguments can be presented for eigenvalue problems of the form $\left(A+\lambda_{1} B_{1}+\cdots+\lambda_{n} B_{n}\right) x=0$.

\section{Linked systems and quadratic eigenvalue problems}

Firstly suppose we are given Hermitean matrices $A_{1}, B_{1}, C_{1}$ of size $n_{1} \times n_{1}$, and $A_{2}$, $B_{2}, C_{2}$ of size $n_{2} \times n_{2}$. Consider the $2 \times 2$ multiparameter eigenvalue problem

$$
\begin{array}{lll}
\left(A_{1}+\lambda_{1} B_{1}+\lambda_{2} C_{1}\right) x_{1}=0, & x_{1} \neq 0, & x_{1} \in \mathbb{R}^{n 1}, \\
\left(A_{2}+\lambda_{1} B_{2}+\lambda_{2} C_{2}\right) x_{2}=0, & x_{2} \neq 0, & x_{2} \in \mathbb{R}^{n_{2}} .
\end{array}
$$

An eigenvalue is a pair $\left(\lambda_{1}, \lambda_{2}\right) \in \mathbb{R}^{2}$ for which this problem can be solved. A customary hypothesis which we shall adopt to ensure the existence of eigenvalues is "right definiteness":

$$
\begin{gathered}
R D: \text { for all } x_{1} \neq 0, x_{2} \neq 0 \\
\operatorname{det}\left|\begin{array}{ll}
\left(B_{1} x_{1}, x_{1}\right) & \left(C_{1} x_{1}, x_{1}\right) \\
\left(B_{2} x_{2}, x_{2}\right) & \left(C_{2} x_{2}, x_{2}\right)
\end{array}\right|>0
\end{gathered}
$$

There is now no loss in assuming that say both $B_{1}$ and $B_{2}$ are positive definite. Under $R D$ there are $n_{1} n_{2}$ eigenvalues $\lambda=\left(\lambda_{1}, \lambda_{2}\right)$ which can be indexed systematically as $\lambda^{(i, j)}$, $1 \leqq i \leqq n_{1}, 1 \leqq j \leqq n_{2}$, in the sense that

$$
W_{k}\left(\lambda^{(i, j)}\right)=A_{k}+\lambda_{1}^{(i, j)} B_{k}+\lambda_{2}^{(i, j)} C_{k}, \quad k=1,2,
$$

has 0 as its $i$ th (respectively $j$ th) eigenvalue for $k=1$ (respectively 2 ). The cone $\hat{C}$ for this situation is 


$$
\hat{C}=\left\{\left(\lambda_{1}, \lambda_{2}\right) \in \mathbb{R}^{2} \mid \lambda_{1}\left(B_{i} x_{i}, x_{i}\right)+\lambda_{2}\left(C_{i} x_{i}, x_{i}\right) \leqq 0 \quad \forall x_{i} \neq 0, i=1,2\right\}
$$

The recent survey paper [3] provides an overview of the (direct) theory of multiparameter eigenvalue problems.

As before we may assume $A_{1}, A_{2}$ have zero leading diagonals.

Theorem 3. Suppose we are given points

$$
s^{(i, j)}=\left(s_{1}^{(i, j)}, s_{2}^{(i, j)}\right) \in \mathbb{R}^{2} \quad 1 \leqq i \leqq n_{1}, \quad 1 \leqq j \leqq n_{2},
$$

with

$$
s^{(1,1)}, \ldots, s^{\left(n_{1}, n_{1}\right)}, s^{\left(n_{1}, n_{1}+1\right)}, \ldots, s^{\left(n_{1}, n_{2}\right)}
$$

ordered by $\hat{C}$-here we have assumed that $n_{1} \leqq n_{2}$. If $s^{(1,1)}, \ldots, s^{\left(n_{1}, n_{1}\right)}$ satisfy Hypothesis 2.2 with respect to $A_{1}, B_{1}, C_{1}$ and $s^{(1,1)}, \ldots, s^{\left(n_{1}, n_{1}\right)}, s^{\left(n_{1}, n_{1}+1\right)}, \ldots, s^{\left(n_{2}, n_{2}\right)}$ satisfy Hypothesis 2.2 with respect to $A_{2}, B_{2}, C_{2}$, then diagonal matrices $D_{1}, D_{2}$ of sizes $n_{1} \times n_{1}$ and $n_{2} \times n_{2}$ respectively can be found so that

$$
\begin{gathered}
s^{(i, i)} \in Z_{i}\left(A_{1}+D_{1}\right) \cap Z_{1}\left(A_{2}+D_{2}\right), \quad 1 \leqq i \leqq n_{1} \\
s^{\left(n_{1}, i\right)} \in Z_{i}\left(A_{2}+D_{2}\right), \quad n_{1}+1 \leqq i \leqq n_{2} .
\end{gathered}
$$

This is parallel to our earlier result [4, Theorem 4.1].

As a further application of our main result we consider the quadratic eigenvalue problem

$$
\left(A+\lambda B+\lambda^{2} C\right) x=0, \quad x \neq 0
$$

where $A, B, C$ are given $n \times n$ symmetric matrices. We can assume that either $B$ or $C$ is positive definite and we ask for conditions under which a diagonal $D$ can be found so that the problem with $A+D$ in place of $A$ has given numbers $s_{1}, \ldots, s_{n}$ as eigenvalues.

Theorem 4. Suppose $\left(s_{i}, s_{i}^{2}\right), 1 \leqq i \leqq n$, are $\hat{C}$-ordered and satisfy Hypothesis 2.2 with respect to $A, B, C$. Then a diagonal $D$ can be found so that the quadratic eigenvalue problem $\left(A+D+\lambda B+\lambda^{2} C\right) x=0$ has $\lambda=s_{1}, \ldots, s_{n}$, as eigenvalues.

The above results answer but a few of the many open questions in inverse eigenvalue theory in the multiparameter setting. Our earlier discussion ([4], Section 6) gave a brief outline of other interesting possibilities.

\section{REFERENCES}

1. E. K. Blum, Numerical Analysis and Computation, Theory and Practice (Addison-Wesley, Reading, Mass., 1972). 
2. P. Binding and P. J. Browne, Spectral properties of two-parameter eigenvalue problems, Proc. Roy. Soc. Edinburgh 89A (1981), 157-173.

3. P. J. BRownE, Multiparameter problems: The last decade, Proceedings 1982 Dundee Conference on Ordinary and Partial Differential Equations (Springer-Verlag Lecture Notes in Mathematics, Vol. 964), 95-109.

4. P. J. Browne and B. D. Steeman, Inverse multiparameter eigenvalue problems for matrices, Proc. Roy. Soc. Edinburgh 100 A (1985), 29-38.

5. K. P. Hadeler, Ein inverses eigenwertproblem, Lin. Alg. and Appns. 1 (1968), 83-101.

6. K. P. Hadeler, Existenz- und eindeutigkeitssatze fur inverse eigenwertaufgaben mit hilfe des topologischen abbildungsgrades, Arch. Rational Mech. Anal. 42 (1971), 317-322.

Department of Mathematics and Statistics

University OF CALgary

Calgary, Alberta

Canada T2N 1 N4
Department of Mathematical Sciences UNIVERSITY OF DUNDEE

DundeE DD1 $4 \mathrm{H} 4$ 